Homology, Homotopy and Applications, vol.15(2), 2013, pp.1-7

\title{
THE GEOMETRIC REALIZATION OF MONOMIAL IDEAL RINGS AND A THEOREM OF TREVISAN
}

\author{
A. BAHRI, M. BENDERSKY, F. R. COHEN AND S. GITLER
}

(communicated by Donald M. Davis)

\begin{abstract}
A direct proof is presented of a form of Alvise Trevisan's theorem [7], that every monomial ideal ring is represented by the cohomology of a topological space. Certain of these rings are shown to be realized by polyhedral products indexed by simplicial complexes.
\end{abstract}

\section{Introduction}

In the paper [7], Alvise Trevisan showed that every ring which is a quotient of an integral polynomial ring with two dimensional generators by an ideal of monomial relations, can be realized as the integral cohomology ring of a topological space. Moreover, he showed that the rings could be all realized with spaces which are generalized Davis-Januszkiewicz spaces. These spaces are colimits over multicomplexes which are generalizations of simplicial complexes.

Here is presented a direct proof of the "realization" part of Trevisan's theorem. It uses a result of Fröberg from [5] which asserts that a map known as "polarization" produces, in a natural way, a regular sequence of degree-two elements. This allows for the realization of any monomial ideal ring by a certain pullback.

It is noted also that certain families of monomial ideal rings, beyond StanleyReisner rings, can be realized as generalized Davis-Januszkiewicz spaces based on ordinary simplicial complexes. Of course, as Trevisan shows, multicomplexes are needed in general.

Through the paper, all cohomology is taken with integral coefficients.

\section{The main result}

Let $\mathbb{Z}\left[x_{1}, \ldots, x_{n}\right]$ be a polynomial ring on generators of degree two and

$$
M=\left\{m_{j}\right\}_{j=1}^{r}, \quad m_{j}=x_{1}^{t_{1 j}} x_{2}^{t_{2 j}} \cdots x_{n}^{t_{n j}}
$$

A. B. was supported in part by a Rider University Summer Research Fellowship and grant number 210386 from the Simons Foundation; F. R. C. was supported partially by DARPA grant number 2006-06918-01.

Received September 19, 2012, revised April 18, 2013; published on July 31, 2013.

2000 Mathematics Subject Classification: Primary: 13F55, Secondary: 55T20, 57T35.

Key words and phrases: monomial ideal ring, Stanley-Reisner ring, Davis-Januszkiewicz space, polarization, polyhedral product.

Article available at http://intlpress.com/HHA/v15/n2/a1 and doi:10.4310/HHA.2013.v15.n2.a1

Copyright (C) 2013, International Press. Permission to copy for private use granted. 
be a set of minimal monomials, that is, no monomial divides another. Here, the exponent $t_{i j}$ might be equal to zero but every $x_{i}$ must appear in some $m_{j}$. Notice that the set $M$ is determined by the $n \times r$ matrix $\left(t_{i j}\right)$. Denote by $I(M)$ the ideal in $\mathbb{Z}\left[x_{1}, \ldots, x_{n}\right]$ generated by the minimal monomials $m_{j}$ and set

$$
A=A(M)=\mathbb{Z}\left[x_{1}, \ldots, x_{n}\right] / I(M)
$$

a monomial ideal ring. From this is defined a second monomial ideal ring $A(\bar{M})$ with monomial ideal generated by square free monomials. For each $i=1,2, \ldots, n$ set

$$
t_{i}=\max \left\{t_{i 1}, t_{i 2}, \ldots, t_{i r}\right\},
$$

the largest entry in the $i$-th row of $\left(t_{i j}\right)$. Next, introduce new variables of degree two $y_{i 1}, y_{i 2}, \ldots, y_{i t_{i}}$ for each $i=1,2, \ldots, n$. For each monomial $m_{j}=x_{1}^{t_{1 j}} x_{2}^{t_{2 j}} \cdots x_{n}^{t_{n j}}$, set

$$
\bar{m}_{j}=\left(y_{11} y_{12} \cdots y_{1 t_{1 j}}\right)\left(y_{21} y_{22} \cdots y_{2 t_{2 j}}\right) \cdots\left(y_{n 1} y_{n 2} \cdots y_{n t_{n j}}\right) .
$$

Let $\bar{M}=\left\{\bar{m}_{j}\right\}_{j=1}^{r}$ and define an algebra $B=B(\bar{M})$ by

$$
B=\mathbb{Z}\left[y_{11}, y_{12}, \ldots, y_{1 t_{1}}, y_{21}, y_{22}, \ldots, y_{2 t_{2}}, \ldots, y_{n 1}, y_{n 2}, \ldots, y_{n t_{n}}\right] / I(\bar{M}) .
$$

The monomials here are square-free so $B$ is a Stanley-Reisner algebra which determines a simplicial complex $K(\bar{M})$. (This process which constructs $B$ from $A$ is known in the literature as polarization.) Associated to this simplicial complex is a fibration

$$
Z\left(K(\bar{M}) ;\left(D^{2}, S^{1}\right)\right) \longrightarrow \mathcal{D} \mathcal{J}(K(\bar{M})) \longrightarrow B T^{d(\bar{M})},
$$

where $d(\bar{M})=\sum_{1=1}^{n} t_{i}$, with $t_{i}$ as in (3), $\mathcal{D} \mathcal{J}(K(\bar{M}))$ is the Davis-Januszkiewicz space of the simplicial complex $K(\bar{M})$, and $Z\left(K(\bar{M}) ;\left(D^{2}, S^{1}\right)\right)$ is the moment-angle complex corresponding to $K(\bar{M})$, [3]. Recall that the Davis-Januszkiewicz space has the property that

$$
H^{*}(\mathcal{D} \mathcal{J}(K(\bar{M}))) \cong B .
$$

Define next a diagonal map $\Delta: T^{n} \longrightarrow T^{d(\bar{M})}$ by

$$
\Delta\left(x_{1}, x_{2}, \ldots, x_{l}\right)=\left(\Delta_{t_{1}}\left(x_{1}\right), \Delta_{t_{2}}\left(x_{2}\right), \ldots, \Delta_{t_{n}}\left(x_{l}\right)\right),
$$

where $\Delta_{t_{i}}\left(x_{i}\right)=\left(x_{i}, x_{i}, \ldots, x_{i}\right) \in T^{t_{i}}$. In the diagram below, let $W(A)$ be defined as the pullback of the fibration.

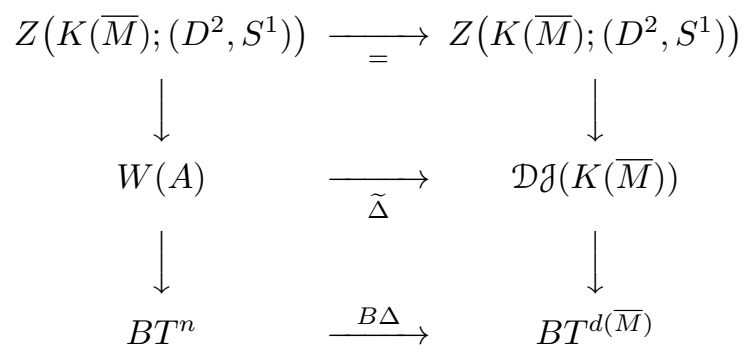


The diagram (8) extends to a larger diagram

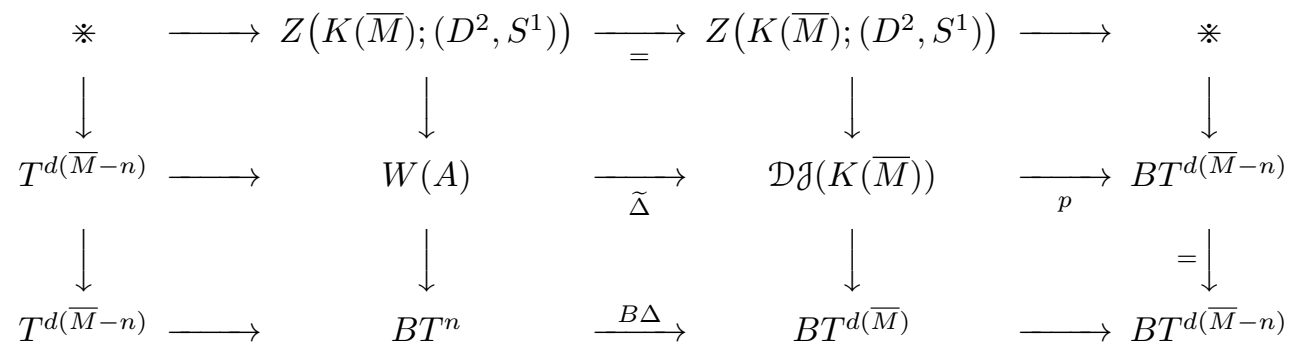

where the fact that $W(A)$ is a pullback implies that

$$
T^{d(\bar{M})-n} \stackrel{p}{\longrightarrow} W(A) \stackrel{\widetilde{\Delta}}{\longrightarrow} \mathcal{D} \mathcal{J}(K(\bar{M}))
$$

is a fibration too. A long exact homotopy sequence argument comparing $W(A)$ to the homotopy fibre of $p$ shows that

$$
W(A) \stackrel{\widetilde{\Delta}}{\longrightarrow} \mathcal{D} \mathcal{J}(K(\bar{M})) \stackrel{p}{\longrightarrow} B T^{d(\bar{M})-n}
$$

is a homotopy fibration. Recall that $d(\bar{M})=\sum_{1=1}^{n} t_{i}$ and choose generators

$$
H^{*}\left(B T^{d(\bar{M})-n}\right) \cong \mathbb{Z}\left[u_{12}, \ldots, u_{1 t_{1}}, u_{22}, \ldots, u_{2 t_{2}}, \ldots, u_{n 2}, \ldots, u_{n t_{n}}\right],
$$

so that

$$
p^{*}\left(u_{i k_{i}}\right)=y_{i 1}-y_{i k_{i}} \quad i=1,2, \ldots, n, \quad k_{i}=2,3, \ldots, t_{i} .
$$

This choice is possible because of the commutativity of the bottom right square in the large diagram above and the description of $H^{*}(\mathcal{D J}(K(\bar{M})))$ given in (5) and (6). Set $\theta_{i k_{i}}:=p^{*}\left(u_{i k_{i}}\right)$. The proposition following is a basic result about the diagonal map $\Delta$ (the polarization map); a proof may be found in [5, page 30$]$.

Proposition 2.1 (Fröberg). Over any field $k$, the sequence $\left\{\theta_{i k_{i}}\right\}$ is a regular sequence of degree-two elements in the ring $H^{*}(\mathcal{D J}(K(\bar{M})) ; k)$.

This result allows for a direct proof of the realization theorem.

Theorem 2.2. There is an isomorphism of rings

$$
H^{*}(W(A) ; \mathbb{Z}) \longrightarrow A(M) .
$$

Proof. Working over a field $k$ and following Masuda-Panov, [6, Lemma 2.1], we use the Eilenberg-Moore spectral sequence associated to the fibration (10). It has

$$
E_{2}^{*, *}=\operatorname{Tor}_{H^{*}\left(B T^{d(\bar{M})-n}\right)}^{*, *}\left(H^{*}(\mathcal{D} \mathcal{J}(K(\bar{M})), k) .\right.
$$

Now $H^{*}(\mathcal{D} \mathcal{J}(K(\bar{M})))$ is free as an $H^{*}\left(B T^{d(\bar{M})-n}\right)$-module by Proposition 2.1, so

$$
\begin{aligned}
\operatorname{Tor}_{H^{*}\left(B T^{d(\bar{M})-n}\right)}^{*, *}\left(H^{*}(\mathcal{D J}(K(\bar{M}))), k\right) & =\operatorname{Tor}_{H^{*}\left(B T^{d(\bar{M})-n}\right)}^{0, *}\left(H^{*}(\mathcal{D J}(K(\bar{M}))), k\right) \\
& =H^{*}(\mathcal{D} \mathcal{J}(K(\bar{M}))) \otimes_{H^{*}\left(B T^{d(\bar{M})-n}\right)} k \\
& =H^{*}(\mathcal{D} \mathcal{J}(K(\bar{M}))) / p^{*}\left(H^{>0}\left(B T^{d(\bar{M})-n}\right)\right) .
\end{aligned}
$$

It follows that the Eilenberg-Moore spectral sequence collapses at the $E_{2}$ term and 
hence, as groups,

$$
H^{*}(W(A))=H^{*}(\mathcal{D} \mathcal{J}(K(\bar{M}))) / p^{*}\left(H^{>0}\left(B T^{d(\bar{M})-n}\right)\right),
$$

from which we conclude that $H^{*}(W(A) ; k)$ is concentrated in even degrees. Taking $k=\mathbb{Q}$ gives the result that in odd degree, $H^{*}(W(A) ; \mathbb{Z})$ consists of torsion only. Unless this torsion is zero, the argument above with $k=\mathbb{F}_{p}$ for an appropriate $p$ implies a contradiction. It follows that $H^{*}(W(A) ; \mathbb{Z})$ is concentrated in even degrees.

Lemma 2.3. The integral Serre spectral sequence of the fibration (10) collapses.

Proof. The spaces in the fibration have integral cohomology concentrated in even degrees.

The $E_{2}$-term of the Serre spectral sequence is

$$
H^{*}(W(A) ; \mathbb{Z}) \otimes H^{*}\left(B T^{d(\bar{M})-n} ; \mathbb{Z}\right) .
$$

It follows that, as a ring, $H^{*}(W(A) ; \mathbb{Z})$ is the quotient of $H^{*}(\mathcal{D} \mathcal{J}(K(\bar{M})))$ by the two-sided ideal $L$ generated by the image of $p^{*}$. So there is an isomorphism of graded rings,

$$
H^{*}(W(A) ; \mathbb{Z}) \longrightarrow H^{*}(\mathcal{D} \mathcal{J}(K(\bar{M}))) / L \cong A(\bar{M}) / L \cong A(M),
$$

completing the proof of Theorem 2.2.

Remark 2.4. The Eilenberg-Moore spectral sequence of the fibration

$$
Z\left(K(\bar{M}) ;\left(D^{2}, S^{1}\right)\right) \longrightarrow W(A) \longrightarrow B T^{n}
$$

collapses and so it can be used to compute the cohomology of $Z\left(K(\bar{M}) ;\left(D^{2}, S^{1}\right)\right)$, the two-connected covering of $W(A)$.

\section{On the geometric realization of certain monomial ideal rings by ordinary polyhedral products}

In this section, polyhedral products, $[\mathbf{1}]$, involving finite and infinite complex projective spaces are used to realize certain classes of monomial ideal rings. As noted earlier, generalizations of the Davis-Januszkiewicz spaces to the realm of multicomplexes are required in order to realize all monomial ideal rings; see Trevisan [7].

The class which can be realized by ordinary polyhedral products is restricted to those monomials

$$
M=\left\{m_{j}\right\}_{j=1}^{r}, \quad m_{j}=x_{1}^{t_{1 j}} x_{2}^{t_{2 j}} \cdots x_{n}^{t_{n j}}
$$

of (1), which satisfy the condition:

* $t_{i j}$ is constant over all monomials $m_{j}$ which have $t_{i j}$ and at least one other exponent both non-zero. 
In particular, a monomial ring of the form

$$
\mathbb{Z}\left[x_{1}, x_{2}, x_{3}\right] /\left\langle x_{1}^{2} x_{2}, x_{1}^{2} x_{3}^{4}, x_{3}^{5}\right\rangle
$$

can be realized by an ordinary polyhedral product. As usual, let $(\underline{X}, \underline{A})$ denote a family of CW pairs

$$
\left\{\left(X_{1}, A_{1}\right),\left(X_{2}, A_{2}\right), \ldots,\left(X_{n}, A_{n}\right)\right\} .
$$

Given a monomial ring $A(M)$ of the form (2), satisfying the condition $*$ above, a simplicial complex $K$ and a family of pairs $(\underline{X}, \underline{A})$ will be specified so that

$$
H^{*}(Z(K ;(\underline{X}, \underline{A})) ; \mathbb{Z})=A(M),
$$

where $Z(K ;(\underline{X}, \underline{A})$ represents a polyhedral product as defined in $[\mathbf{1}]$.

Construction 3.1. Let $K$ be the simplicial complex on $n$ vertices $\left\{v_{1}, v_{2}, \ldots, v_{n}\right\}$ which has a minimal non-face corresponding to each $m_{i}$ having at least two non-zero exponents. If $m_{i}$ has non-zero exponents

$$
t_{j_{1} i}, t_{j_{2} i}, \ldots, t_{j_{t} i}
$$

then $K$ will have a corresponding minimal non-face $\left\{v_{j_{1}}, v_{j_{2}}, \ldots, v_{j_{t}}\right\}$. Moreover, these will be the only minimal non-faces of $K$.

For example, the ring (11) above will have associated to it the simplicial complex $K$ on vertices $\left\{v_{1}, v_{2}, v_{3}\right\}$ and will have minimal non-faces $\left\{v_{1}, v_{2}\right\}$ and $\left\{v_{1}, v_{3}\right\}$. So, $K$ will be the disjoint union of a point and a one-simplex.

For the set of monomials $M$ satisfying condition $*$, the cases following are distinguished in terms of (1) for fixed $i \in\{1,2, \ldots, n\}$.

1. For certain $j, t_{i j}=1, t_{i^{\prime} j} \neq 0$ for some $i^{\prime} \neq i$ and $t_{i k}=0$ otherwise.

2. For certain $j, t_{i j}=q_{i}>1, t_{i^{\prime} j} \neq 0$ for some $i^{\prime} \neq i$ and $t_{i k}=0$ otherwise.

3. $m_{j}=x_{i}^{s_{i}}$ for some $j$ and $t_{i k}=0$ for $k \neq j$.

4. $m_{j}=x_{i}^{s_{i}}$ for some $j$ and if $t_{i k} \neq 0$ for $k \neq j$, then $t_{i k}=q_{i}<s_{i}$.

With this classification in mind, define a family of CW-pairs

$$
(\underline{X}, \underline{A})=\left\{\left(X_{i}, A_{i}\right): i=1, \ldots, n\right\}
$$

by

$$
\left(X_{i}, A_{i}\right)= \begin{cases}\left(\mathbb{C} P^{\infty}, *\right) & \text { if } i \text { satisfies (1) } \\ \left(\mathbb{C} P^{\infty}, \mathbb{C} P^{q_{i}-1}\right) & \text { if } i \text { satisfies (2), } \\ \left(\mathbb{C} P^{s_{i}-1}, *\right) & \text { if } i \text { satisfies (3), } \\ \left(\mathbb{C} P^{s_{i}-1}, \mathbb{C} P^{q_{i}-1}\right) & \text { if } i \text { satisfies (4). }\end{cases}
$$

The next theorem describes the polyhedral products which have cohomology realizing the monomial ideal rings satisfying condition $*$.

Theorem 3.2. Let $A(M)$ be a monomial ring of the form (2), satisfying the condition * and $K$, the simplicial complex defined by Construction 3.1, then

$$
H^{*}(Z(K ;(\underline{X}, \underline{A})) ; \mathbb{Z})=A(M)
$$

where $(X, A)$ is the pair specified by (12). 
Remark 3.3. The improvement here over [2, Theorem 10.5] consists of the inclusion of cases (3) and (4) above. The polyhedral products which realize the monomial ideal rings discussed in [1] have $X_{i}=\mathbb{C} P^{\infty}$ for all $i=1,2, \ldots, n$.

Proof of Theorem 3.2. Set $Q=\left(q_{1}, q_{2}, \ldots, q_{n}\right)$ with $q_{i} \geqslant 1$ for all $i$ and write the spaces $A_{i}$ of (12) as $\mathbb{C} P^{q_{i}-1}$ where $q_{i}=1$ if $A_{i}=*$, a point. Write

$$
(\underline{X}, \underline{A})=\left(\underline{X}, \underline{\mathbb{C}}^{Q-1}\right)=\left\{\left(X_{i}, \mathbb{C} P^{q_{i}-1}\right): i=1,2, \ldots, n\right\}
$$

and consider the commutative diagram

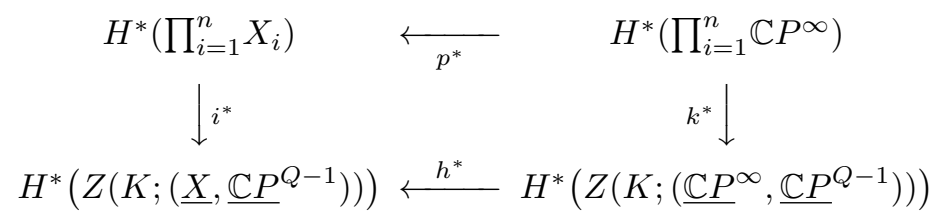

induced by the various inclusion maps. According to [2, Theorem 10.5], there is an isomorphism of rings

$$
H^{*}\left(Z\left(K ;\left(\underline{\mathbb{C} P}^{\infty}, \underline{\mathbb{C}}^{Q-1}\right)\right)\right) \longrightarrow \mathbb{Z}\left[x_{1}, \ldots, x_{n}\right] / I\left(M^{Q}\right),
$$

where $I\left(M^{Q}\right)$ is the ideal generated by all monomials $x_{i_{1}}^{q_{i_{1}}}, x_{i_{2}}^{q_{i_{2}}}, \ldots, x_{i_{k}}^{q_{i_{k}}}$ corresponding to the minimal non-faces $\left\{v_{i_{1}}, v_{i_{2}}, \ldots, v_{i_{k}}\right\}$ of $K$. Moreover, the proof of [2, Lemma 10.3] shows that the composition $i^{*} p^{*}$ is a surjection. The commutativity of diagram (13) implies that these relations all hold in $H^{*}\left(Z\left(K ;\left(\underline{X}, \underline{\mathbb{C}}^{Q-1}\right)\right)\right)$. In addition to these, the relation $x_{i}^{s_{i}}=0$ is included for each $i$ satisfying $X_{i}=\mathbb{C} P^{s_{i}-1}$. These relations account for all the relations determined by $I(M)$. The remainder of the argument shows that $I(M)$ determines all relations in $H^{*}(Z(K ;(\underline{X}, \underline{A})) ; \mathbb{Z})$. Consider now the space

$$
W_{k}=\mathbb{C} P^{q_{1}-1} \times \cdots \times \mathbb{C} P^{q_{k-1}-1} \times X_{k} \times \mathbb{C} P^{q_{k+1}-1} \times \cdots \times \mathbb{C} P^{q_{n}-1}
$$

corresponding to the simplex $\left\{v_{k}\right\} \in K$, consisting of a single vertex. The composition

$$
W_{k} \longrightarrow Z\left(K ;\left(\underline{X}, \underline{\mathbb{C} P}^{Q-1}\right)\right) \longrightarrow \prod_{i=1}^{n} X_{i}
$$

factors the natural inclusion $W_{k} \longrightarrow \prod_{i=1}^{n} X_{i}$. From this observation follows the fact that no other monomial relations occur in $H^{*}\left(Z\left(K ;\left(\underline{X}, \underline{\mathbb{C} P^{Q-1}}\right)\right)\right)$ other than those determined by $I(M)$. Suppose next that there is a linear relationship of the form

$$
a \omega=\sum_{i=1}^{k} a_{i} \omega_{i},
$$

where $a, a_{i} \in \mathbb{Z}$ and $\omega, \omega_{i}$ are monomials in the $x_{i}, i=1,2, \ldots, n$. Without loss of generality, $\omega$ and $\omega_{i}$ can be assumed to be not divisible by any of the monomials in $M$. Suppose $\omega=x_{j_{1}}^{\lambda_{1}} x_{j_{2}}^{\lambda_{2}} \cdots x_{j_{l}}^{\lambda_{l}}$, then $\sigma=\left\{v_{j_{1}}, v_{j_{2}}, \ldots, v_{j_{l}}\right\} \in K$ is a simplex and so is a full subcomplex of $K$. (The corresponding polyhedral product $Z\left(\sigma ;\left(\underline{X}, \underline{\mathbb{C}}^{Q-1}\right)\right)$ is a product of finite and infinite complex projective spaces.) This implies, by $[\mathbf{4}$, Lemma 2.2.3], that $H^{*}\left(Z\left(\sigma ;\left(\underline{X}, \underline{\mathbb{C}}^{Q-1}\right)\right)\right)$ must be a direct summand in $H^{*}(Z(K$; $\left.\left.\left(\underline{X}, \underline{\mathbb{C}}^{Q-1}\right)\right)\right)$ contradicting the relation (14). 


\section{References}

[1] A. Bahri, M. Bendersky, F. R. Cohen and S. Gitler, The Polyhedral Product Functor: a method of computation for moment-angle complexes, arrangements and related spaces, Advances in Mathematics 225 (2010), 1634-1668.

[2] A. Bahri, M. Bendersky, F. R. Cohen and S. Gitler, Operations on polyhedral products and a new topological construction of infinite families of toric manifolds. Online at: http://arxiv.org/abs/1011.0094.

[3] V. Buchstaber and T. Panov, Torus actions and their applications in topology and combinatorics, AMS University Lecture Series 24 (2002).

[4] G. Denham and A. Suciu, Moment-angle complexes, monomial ideals and Massey products, Pure and Applied Mathematics Quarterly 3, no. 1 (2007), 25-60.

[5] R. Fröberg, A study of graded extremal rings and of monomial rings, Math. Scand. 51 (1982), 22-34.

[6] M. Masuda and T. Panov On the cohomology of torus manifolds. Available at: http://arxiv.org/abs/math/0306100.

[7] A. J. Trevisan, Generalized Davis-Januszkiewicz spaces, multicomplexes and monomial rings, Homology, Homotopy and Applications 13, no. 1 (2011), 205221.

A. Bahri bahri@rider.edu

Department of Mathematics, Rider University, Lawrenceville, NJ 08648, USA

M. Bendersky mbenders@hunter.cuny.edu

Department of Mathematics, Hunter College, East 695 Park Avenue, New York, NY 10065, USA

F. R. Cohen cohf@math.rochester.edu

Department of Mathematics, University of Rochester, Rochester, NY 14625, USA

S. Gitler sgitler@math.cinvestav.mx

El Colegio Nacional, Gonzalez Obregon 24 C, Centro Historico, Mexico City, Mexico. 